\title{
Preparation of starch stabilized silver nanoparticles with spatial self-phase modulation properties by laser ablation technique.
}

\begin{abstract}
Silver nanoparticles inside the starch solution have been successfully fabricated by laser ablation of a silver plate immersed in starch solution. The ablation has been done using a Qswitched Nd:YAG laser at $10 \mathrm{~Hz}$ repetition rate. The starch solution allows for the formation of silver nanoparticles with uniform particle diameters and well dispersed. The ablation was performed at different time durations to study the influence of the laser ablation time on efficiency of particle formation and sizes. The Spatial Self-phase modulation phenomena which can determine the nonlinear optical property of the samples were also investigated for starch solutions containing silver nanoparticles.
\end{abstract}

Keyword: Starch; Silver nanoparticles; Laser ablation. 